EGU2020-18758

https://doi.org/10.5194/egusphere-egu2020-18758

EGU General Assembly 2020

(c) Author(s) 2021. This work is distributed under

the Creative Commons Attribution 4.0 License.

\title{
Deciphering compositional processes in inner airless bodies of our Solar System
}

Francesca Zambon ${ }^{1}$, Federico Tosi ${ }^{1}$, Sébastien Besse ${ }^{2}$, Rosario Brunetto ${ }^{3}$, Cristian Carli ${ }^{1}$, JeanPhilippe Combe ${ }^{4}$, Olivier Forni ${ }^{5}$, Rachel Klima ${ }^{6}$, Katrin Krohn ${ }^{7}$, David Rothery ${ }^{8}$, Katrin Stephan ${ }^{7}$, Kerri Donaldson-Hanna ${ }^{9}$, Oceane Barraud ${ }^{10,11}$, and Jacopo Nava ${ }^{12}$

'INAF - IAPS, Via del Fosso del Cavaliere, 100, I-00133 Rome, Italy (francesca.zambon@inaf.it)

${ }^{2}$ ESA/ESAC, Villafranca del Castillo, 28692 Villanueva de la Cañada, Madrid, Spain

${ }^{3}$ Institut d'Astrophysique Spatiale (IAS), Campus d'Orsay, Orsay 91405, France

${ }^{4}$ Bear Fight Institute, 22 Fiddler's Road, Winthrop WA 98862, USA

${ }^{5}$ IRAP-CNRS, Université de Toulouse 9, av. du Colonel Roche, F-31028 Toulouse, France

${ }^{6} J$ ohns Hopkins University Applied Physics Laboratory, 11100 Johns Hopkins Rd., Laurel MD 20723, USA

${ }^{7}$ Institute of Planetary Research (DLR), Rutherfordstrasse 2, D-12489 Berlin, Germany

${ }^{8}$ The Open University, Walton Hall, Milton Keynes, MK7 6AA, UK

${ }^{9}$ University of Central Florida , 4111 Libra Drive, Physical Sciences Building (PSB) 430 ,Orlando, Florida 32816-2385

${ }^{10}$ University of Paris Sciences et Lettres (PSL), Laboratoire d'études spatiales et d'instrumentation en astrophysique (LESIA)

${ }^{11}$ Observatoire de Paris-Meudon, 5 Place Jules Janssen, 92190 Meudon, France

${ }^{12}$ University of Padua, Department of Geoscience, Via Gradenigo 6, I-35131 Padua, Italy

Over the last decades, the exploration of our Solar System carried out by automatic probes allowed a huge leap in our understanding of the planets, their main satellites and minor bodies such as asteroids and comets. However, despite the large number of diverse datasets available nowadays, comparative studies of different bodies are still poorly addressed in several cases, in particular for airless bodies.

The primary goal of our two-year project, selected in the framework of the "ISSI/ISSI-BJ Joint Call for Proposals 2019 for International Teams in Space and Earth Sciences", is to quantify similarities and differences in the surface mineralogy of Vesta, Mercury and the Moon, substantially enhancing the scientific return of individual instrumental datasets and/or individual space missions. Here, we give an overview of our project, we clarify what is the status after the first team meeting held in March 2020.

Our project focuses on two specific questions:

Our overall approach is to apply various techniques of analysis on hyper- and multispectral data sets that are publicly available, such as those on acquired by the Dawn mission at Vesta, MESSENGER datasets obtained at Mercury and Chandrayaan-1 data for the Moon.

This work is supported by the International Space Science Institute (ISSI) and by INAF-IAPS. 
